Open Access

\title{
Application of Adomian decomposition method to free vibration analysis of thin isotropic rectangular plates submerged in fluid
}

\author{
Gbeminiyi M. Sobamowo', Saheed A. Salawu ${ }^{2 *}$ and Ahmed A. Yinusa ${ }^{1}$
}

\author{
*Correspondence: \\ safolu@outlook.com \\ ${ }^{2}$ Department of civil and \\ Environmental Engineering, \\ University of Lagos, Akoka, Nigeria \\ Full list of author information is \\ available at the end of the article
}

\begin{abstract}
Investigating dynamic free vibration response of isotropic thin rectangular plate resting on two-parameter elastic foundation is of interest in the field of geotechnics, structure, highway, railway and mechanical engineering. This study employs analytical approach to the investigation of free vibration analysis of isotropic rectangular plate submerged in fluid and resting on combined elastic foundations under different support conditions. The nonlinear partial differential equation of the system is transformed into nonlinear ordinary differential equation using the Galerkin method of separation. The resulting ordinary differential equation is solved using Adomian decomposition method (ADM). The reliability of the solutions obtained is validated with numerical and existing results as reported in the literature. Also, the bending moment and stress are analysed. The analytical solutions are used for the investigation of dynamic behaviour of plates in fluid, effect of aspect ratio, effect of elastic foundation parameters on natural frequency and effect of bending moment and stress on the mode of the vibrating plate. From the results, it is observed that the increase in elastic foundation parameter increases the natural frequency. Increasing the aspect ratio increases the natural frequency. Increasing the combined elastic foundation parameters increases the natural frequency. Natural frequency of plates reduces when submerged in fluid with the mode shapes not significantly affected. The node and antinodes of the mode shapes are affected by moment and stress. It is expected that the present study will add to the existing knowledge in the field of vibration of plates.
\end{abstract}

Keywords: Dynamic analysis, Rectangular plate, Deflection, Winkler and Pasternak, Adomian decomposition method

\section{Introduction}

Recently, interest has arose into the study of dynamic behaviour of thin rectangular plate. This is because plates are considered an important structural member due to their wide acceptable usage in various fields of engineering. This is also due to their light weight and high strength. Therefore, the study and analyses of natural frequency and modal behaviour are justified. In the study of free vibration analysis of thin rectangular plate on elastic foundation, Motaghian and Akin [1] used an exact method in obtaining the solution. In another study, Rezaiefar and Galal [2] worked on free vibration of rectangular

(C) The Author(s). 2020 Open Access This article is licensed under a Creative Commons Attribution 4.0 International License which permits use, sharing, adaptation, distribution and reproduction in any medium or format, as long as you give appropriate credit to the original author(s) and the source, provide a link to the Creative Commons licence, and indicate if changes were made. The images or other third party material in this article are included in the article's Creative Commons licence, unless indicated otherwise in a credit line to the material. If material is not included in the article's Creative Commons licence and your intended use is not permitted by statutory regulation or exceeds the permitted use, you will need to obtain permission directly from the copyright holder. To view a copy of this licence, visit http://creativecommons.org/licenses/by/4.0/. 
plate with nonlinear load using finite element method. The adoption of two-parameter foundation has proven to be more reliable than only Winkler foundation due to the ability to take care of the shear interaction among spring element. However, Shariyat and Alipour [3] used a semi-analytical method of solution to investigate the free vibration analysis of functionally graded plate on two-parameter elastic foundation. Meanwhile, Benferhat et al. [4] determine porosity effect on free vibration analysis and bending of functionally graded plate resting on two-parameter foundation. Much attention is given to plates under fluidic interaction due to the importance and application in ship building, marine, nuclear and ocean engineering. The study of plate-fluid interaction in engineering is justified for safety and design purpose. Many literatures had studied the characteristic of immersed and submerged plate in fluids. Lamb [5] carried out an analytical approach into the investigation of fluid-plate coupled system. He determined the natural frequency of clamped circular plate in contact with water using Rayleigh's method; however, Sobamowo et al. [6] investigated plates in a porous medium. In another work, Kwak [7] determined the added virtual mass incremental (AVMI) factor which ascertained the fact that the increase in inertial is due to the presence of fluid in plates. Further study, Soedel and Soedel [8] established a coupled equation of motion of plate carrying fluid. Kerboua et al. [9] later developed a mathematical model for free vibration of plates in contact with fluid using the finite element method. In a later work, Ozdemir [10] employed the finite element method in the vibration response of rectangular Mindlin plate on the Winkler foundation. Also, Mohd et al. [11] performed a numerical analysis on semi-empirical relations. Nikbakhat and Behnamfar [12] demonstrated an experiment on the structures under subway-induced vibrations. In recent time, researches have proposed and applied several semi-analytical methods in analysing dynamic behaviour of rectangular plate resting on nonlinear foundations. Bayat et al. [13] applied the homotopy perturbation method (HPM) for nonlinear free vibration of tapered beams. Werfalli and Aburuga [14] used the Galerkin method for the analysis of rectangular plate. Analytical method [15-18] has proven to be the most reliable method of solution when compared to others like numerical and semi-analytical, but the shortcomings of handling nonlinear problem have not been overcome. Adomian and Rach [19] proposed the method of Adomian decomposition method (ADM) in the beginning of 1980. The aim of ADM polynomial is to weaken the nonlinear terms in the governing equation into a series function. Unlike VIM and HPM that have shortcomings of determination of Lagrange multiplier and small parameters, ADM gives a better solution with fast rate of convergence coupled with high accuracy and minimum calculation because discretization of variables or perturbation is not required: ability to solve nonlinear problems without linearization nor use assumptions of weak nonlinearity. Some related works on ADM are [20-27]. Based on the review of the subject, the topic, analytical approach to investigation of free vibration analysis of isotropic rectangular plate submerged in fluid and resting on combined elastic foundations using ADM has not been investigated. Therefore, the present study focuses on the application of ADM to determine dynamic analysis of isotropic rectangular plates resting on Winkler and Pasternak foundations. The solutions are used for parametric studies.

\section{Problem formulation and mathematical analysis}

The present study considers a thin rectangular plate of uniform thickness resting on linear and nonlinear Winkler foundation and Pasternak foundation under different edge 
conditions. The boundary edge of the plate may be free, clamped, simply supported or combination of them as shown in Fig. 1. The following assumptions according to Leissa [28] and Chakraverty [29] are made for the development of the governing equation:

1. Normal to the undeformed mid-surface remains straight and normal to the deformed mid-surface with the same length.

2. The thickness of plate is smaller compared to the other dimensions.

3. Rotary inertia and shear deformation effect are negligible.

4. Normal stresses $\sigma_{z}$ in the transverse direction to the plate are considered negligibly small.

Similarly, the following assumptions are considered for fluid pressure $\Delta p$ [9]:

1. Vibration is considered linear; plate is of uniform density and thickness.

2. Flow of fluid is potential, irrotational and homogenous.

3. Fluid is incompressible.

4. Fluid pressure is normal to the plate surface; shear force is ignored because the flow is inviscid.

5. The motion is considered small.

6. The effect of water dynamic loading has an insignificant effect on mode shapes.

7. The system is conservative.

$$
\begin{aligned}
& \frac{\partial^{4} W(x, y)}{\partial X^{4}}+2 \lambda^{2} \frac{\partial^{4} W(x, y)}{\partial X^{2} \partial Y^{2}}+\lambda^{4} \frac{\partial^{4} W(x, y)}{\partial Y^{4}}-\left(\Omega^{2}+m_{a d d}-K_{L}\right) W(x, y) \\
& -K_{N L} W^{3}(x, y)-g_{s} \frac{\partial^{2} W(x, y)}{\partial X^{2}}=0,
\end{aligned}
$$

The governing equation of the thin plate resting linear and nonlinear Winkler and Pasternak foundations also fluid-interaction in dimensionless form is presented in Eq. (1). $w$ is the transverse deflection, where, $\Omega, g_{s}$ and $K_{\mathrm{L}}$ and $K_{\mathrm{NL}}$ are the dimensionless natural frequency, dimensionless Pasternak's Shear stiffness and dimensionless Winkler's normal stiffness and nonlinear Winkler's, respectively.

\section{Application of Galerkin method of dimensionless governing equation}

Assuming the two opposite edges $Y=0$ and $Y=1$ to be simply supported, deflection function can be represented as follows:

$$
W=W(X) \sin (m \pi Y),
$$

Substitute Eq. (2) into governing differential Eq. (1), we get [22]:

$$
\frac{d^{4} W(X)}{d X^{4}}-2 \lambda^{2} m^{2} \pi^{2} \frac{d^{2} W(X)}{d X^{2}}-\left(\Omega^{2}+M_{\mathrm{add}}-K_{L}-\lambda^{4} m^{4} \pi^{4}\right) W(X)-K_{\mathrm{NL}} W^{3}(X)-g_{s} \frac{d^{2} W(X)}{d X^{2}}=0,
$$

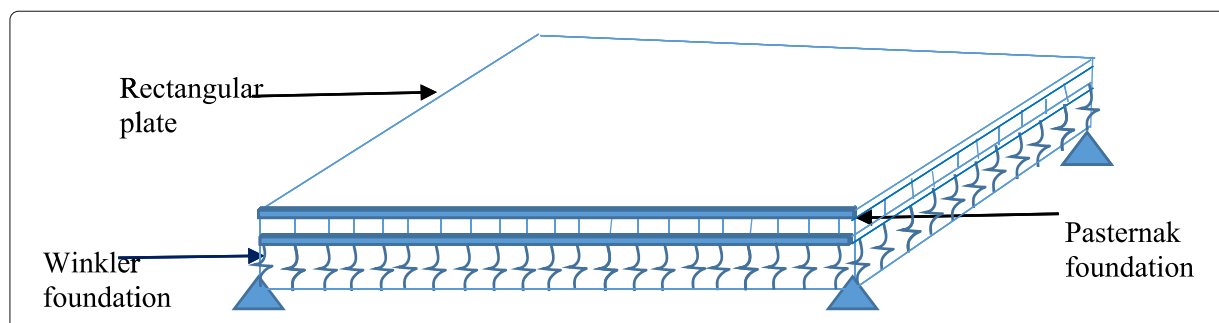

Fig. 1 Showing rectangular plate resting on the Two-Parameter foundation 


\section{Boundary conditions}

Three boundary conditions are considered at $x=0$ and $x=1$, notation are used for brevity sake, SS denotes simply supported at both edges of the rectangular plate, SC means simply supported and clamped at the other ans SF denotes simply supported and free edge support. Classical plate theory defines the dimensionless support conditions as follows:

Clamped edge:

$$
W=\frac{d W}{d X}=0,
$$

Simply supported edge:

$$
W=\frac{d^{2} W}{d X^{2}}-v\left(m^{2} \pi^{2} \lambda^{2}\right) W=0,
$$

Free edge:

$$
\frac{d^{2} W}{d X^{2}}-v\left(m^{2} \pi^{2} \lambda^{2}\right) W=0, \quad \frac{d^{3} W}{d X^{3}}-(2-v)\left(m^{2} \pi^{2} \lambda^{2}\right) \frac{d W}{d X}=0,
$$

where $m$ is an integer, and $v$ is the Poisson ratio.

\section{Method of solution: Adomian decomposition method}

\section{Description of Adomian decomposition method}

The Adomian decomposition method (ADM) is developed by George Adomian in 1990s. It is a semi-analytical method of solving partial and ordinary nonlinear equation. It uses 'Adomian polynomials' for fast convergence of nonlinear aspect of differential equation. The principle of operation is as follows:

Considering the following equation:

$$
L w+N w+R w=g(x)
$$

where $L$ is a linear operator, $N$ is the nonlinear operator, $R$ is the remaining linear operator and $g$ is the inhomogeneous term. If $L$ is the fourth order operator, it is defined by:

$$
L=\frac{d^{4}}{d x^{4}}
$$

Assuming $L$ is invertible, then inverse operator $L^{-1}$ is given as:

$$
L^{-1}(.)=\int_{0}^{x} \int_{0}^{x} \int_{0}^{x} \int_{0}^{x}(.) d x d x d x d x
$$

Therefore,

$$
L^{-1} L w=w(x)-w(0)-x w^{\prime}(0)-\frac{1}{2 !} x^{2} w^{\prime \prime}(0)-\frac{1}{3 !} x^{3} w^{\prime \prime \prime}(0),
$$

Applying $L^{-1}$ to both sides Eq. (7) gives:

$$
w=\Phi_{0}-L^{-1} R w-L^{-1} N w+L^{-1} g(x),
$$

where

$$
\Phi_{0}=\left\{\begin{array}{lr}
w(0), & \text { if } L=\frac{d}{d x} \\
w(0)+x w^{\prime}(0), & \text { if } L=\frac{d^{2}}{d x^{2}} \\
w(0)+x w^{\prime}(0)+\frac{x^{2}}{2 !} w^{\prime \prime}(0), & \text { if } L=\frac{d^{3}}{d x^{3}} \\
w(0)+x w^{\prime}(0)+\frac{x^{2}}{2 !} w^{\prime \prime}(0)+\frac{x^{3}}{3 !} w^{\prime \prime \prime}(0), & \text { if } L=\frac{d^{4}}{d x^{4}}
\end{array}\right.
$$


The decomposition principle comprises decomposing the solution to the sum of infinite number of terms defined by the series:

$$
w=\sum_{n=0}^{\infty} w_{n},
$$

The nonlinear term is written as:

$$
N(w)=\sum_{n=0}^{\infty} A_{n}
$$

where $A_{n}^{\prime} s$ are the Adomian polynomials.

$$
A_{n}=\frac{1}{n !}\left[\frac{\partial^{n}}{\partial \xi^{n}}\left(\left(\sum_{i=0}^{\infty} \xi^{i} w_{i}(x)\right)\right)\right]_{\xi=0} n=0,1,2,3 \ldots ;
$$

where $\xi$ is a grouping parameter.

$$
\begin{aligned}
& A_{0}=N\left(w_{0}\right), \\
& A_{1}=N\left(w_{0}\right) w_{1}, \\
& A_{2}=N^{\prime}\left(w_{0}\right) w_{2}+\frac{1}{2 !} N^{\prime \prime}\left(w_{0}\right) w_{1}^{2},
\end{aligned}
$$

Substituting Eqs. (13) and (14) into (7) gives:

$$
\sum_{n=0}^{\infty} w_{n}=\Phi_{0}-L^{-1} R\left(\sum_{n=0}^{\infty} w_{n}\right)-L^{-1}\left(\sum_{n=0}^{\infty} A_{n}\right)+L^{-1} g(x),
$$

The iterative schemes are:

$$
\begin{aligned}
& w=\Phi_{0}+L^{-1} g(x), \\
& w_{n+1}=-L^{-1} R w_{n}-L^{-1} A_{n},
\end{aligned}
$$

where $n \geq 0$

This results to:

$$
\begin{aligned}
& w_{0}=\Phi_{0}+L^{-1} g(x), \\
& w_{1}=-L^{-1} R w_{0}-L^{-1} A_{0}, \\
& w_{2}=-L^{-1} R w_{1}-L^{-1} A_{1},
\end{aligned}
$$

where $n \geq 0$

\section{Application of ADM to the solution of nonlinear equation under investigation}

According to basic rule of ADM, Eq. (3) is written as:

$$
w_{n}(x)=w(0)+w^{\prime}(0) x+w^{\prime \prime}(0) \frac{x^{2}}{2 !}+w^{\prime \prime \prime}(0) \frac{x^{3}}{3 !}-L^{-1}[R w]-L^{-1}[N w]+L^{-1}[g(x)],
$$

Applying Eq. (5) into Eq. (21), we have:

$$
\begin{aligned}
& w_{n}(x)=w^{\prime}(0) x+w^{\prime \prime \prime}(0) \frac{x^{3}}{3 !} \\
& -L^{-1}\left[2 m^{2} \pi^{2} \lambda^{2} \frac{\mathrm{d}^{2} w}{\mathrm{~d} x^{2}}-\left(\Omega^{2}+M_{\mathrm{add}}-k_{\mathrm{L}}-\lambda^{4} m^{4} \pi^{4}\right) w-g_{s} \frac{d^{2} w}{d x^{2}}\right]-L^{-1}\left[k_{\mathrm{NL}} w^{3}\right],
\end{aligned}
$$


$w^{\prime}(0)$ and $w^{\prime \prime \prime}(0)$ are unknown which are represented by $B$ and $C$, respectively:

$$
\begin{aligned}
& w_{n}(x)=B x+\frac{C x^{3}}{3 !}-L^{-1}\left[2 m^{2} \pi^{2} \lambda^{2} \frac{d^{2} w}{d x^{2}}-\left(\Omega^{2}+M_{\mathrm{add}}-k_{\mathrm{L}}-\lambda^{4} m^{4} \pi^{4}\right) w-g_{s} \frac{d^{2} w}{d x^{2}}\right] \\
& -L^{-1}\left[k_{\mathrm{NL}} w^{3}\right],
\end{aligned}
$$

In Adomian's polynomial, $A^{\prime} s$ are expressed as:

$$
\begin{aligned}
& A_{0}=\left(w_{0}\right)^{3} ; \\
& A_{1}=3\left(w_{0}\right)^{2} w_{1} ; \\
& A_{2}=3\left(w_{0}\right)^{2} w_{2}+3 w_{0}\left(w_{1}\right)^{2} ; \\
& A_{3}=6 w_{0} w_{1} w_{2}+\left(w_{1}\right)^{3}+3\left(w_{0}\right)^{2} w_{3} ; \\
& A_{4}=6 w_{0} w_{1} w_{3}+3 w_{0}\left(w_{2}\right)^{2}+3\left(w_{1}\right)^{2} w_{2}+3\left(w_{0}\right)^{2} w_{4} ;
\end{aligned}
$$

The other polynomials are generated in similar way as Eq. (24).

$$
\begin{aligned}
& w_{0}=B x+\frac{1}{6} C x^{3} ; \\
& w_{1}=-L^{-1}\left[2 m^{2} \pi^{2} \lambda^{2} \frac{d^{2} w_{0}}{d x^{2}}-\left(\Omega^{2}+M_{a d d}-k_{L}-\lambda^{4} m^{4} \pi^{4}\right) w_{0}-g_{s} \frac{d^{2} w_{0}}{d x^{2}}\right] \\
& -L^{-1}\left[k_{N L} A_{0}\right], \\
& w_{2}=-L^{-1}\left[2 m^{2} \pi^{2} \lambda^{2} \frac{d^{2} w_{1}}{d x^{2}}-\left(\Omega^{2}+M_{a d d}-k_{L}-\lambda^{4} m^{4} \pi^{4}\right) w_{1}-g_{s} \frac{d^{2} w_{1}}{d x^{2}}\right] \\
& -L^{-1}\left[k_{N L} A_{1}\right], \\
& w_{3}=-L^{-1}\left[2 m^{2} \pi^{2} \lambda^{2} \frac{d^{2} w_{2}}{d x^{2}}-\left(\Omega^{2}+M_{a d d}-k_{L}-\lambda^{4} m^{4} \pi^{4}\right) w_{2}-g_{s} \frac{d^{2} w_{2}}{d x^{2}}\right] \\
& -L^{-1}\left[k_{N L} A_{2}\right],
\end{aligned}
$$

Using the definition (13), the analytical series solution is obtained.

$$
W(x)=\sum_{m=0}^{\infty} W_{m}(x)=W_{0}(x)+W_{1}(x)+W_{2}(x)+\ldots ;
$$

where the constant $B$ and $C$ are found using Eqs. (4), (5) and (6). Setting the controlling parameters to 0 then substitute the values into Eq. (29) lead to the following simultaneous expression:

$$
\begin{aligned}
& \psi_{11}^{(n)}(\Omega) w_{0}+\psi_{12}^{(n)}(\Omega) w_{2}=0 \\
& \psi_{21}^{(n)}(\Omega) w_{0}+\psi_{22}^{(n)}(\Omega) w_{2}=0
\end{aligned}
$$

The polynomials $\psi_{11}, \psi_{12}, \psi_{21}$ and $\psi_{22}$ are represented in terms of the natural frequency $\Omega$. Meanwhile, $\psi_{11}, \psi_{12}, \psi_{21}$ and $\psi_{22}$ are representing a series expression obtained from Eq. (29). Therefore, Eq. (30) may be written in matrix form as:

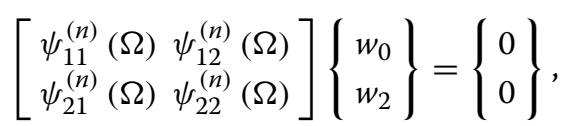

The following characteristic determinant is obtained applying the non-trivial condition:

$$
\left[\begin{array}{lll}
\psi_{11}^{(n)} & (\Omega) & \psi_{12}^{(n)}(\Omega) \\
\psi_{21}^{(n)} & (\Omega) & \psi_{22}^{(n)}(\Omega)
\end{array}\right]=0,
$$


Solving Eq. (32) gives the natural frequencies.

$$
\frac{\left|\Omega_{j}^{(i)}-\Omega_{j}^{(i-1)}\right|}{\left|\Omega_{j}^{(i)}\right|} \leq \varepsilon, j=1,2,3, \ldots n
$$

where the iteration counter is represented by $i$, the estimated value of the $j$ th dimensionless natural frequency is $\Omega_{j}^{(i)}$ and small number chosen is $\varepsilon$. For this study, $\varepsilon=0.0001$. The results show that the use of only few iterations gives a convergent solution.

The deflection series solution of the governing equation for SF boundary condition is:

$$
\begin{aligned}
& W(x)=x-\frac{8367 x^{3}}{14521}+\frac{24211 x^{5}}{75362}-\frac{8315 x^{7}}{314432}+\frac{1515 x^{9}}{369907}-\frac{23 x^{11}}{x^{23}}+\frac{107 x^{13}}{178680}+\frac{5 x^{15}}{11628921} x^{25}-\frac{17}{33008094} \\
& +\frac{4 x^{17}}{644113311}-\frac{x^{19}}{x^{27}}+\frac{x^{21}}{15929564220}+\frac{x^{23}}{599979099008}-\frac{x^{29}}{87813673294953}+\frac{x^{13}}{4724940354278380} \\
& -\frac{x^{2}}{959420400780756366}+\frac{x^{33}}{69863109901089499689}-\frac{x}{18793443763260581094762} \\
& +\frac{x^{3}}{1779726318335411523907948}-\frac{x^{35}}{612598163066578778270048320}+\cdots \cdots
\end{aligned}
$$

\section{Modified of Adomian decomposition method procedure}

The small domain limitation of semi-analytical method has been overcome by the introduction of an after treatment method in power series method. Laplace-Padè approximant has proven to be a very reliable approach that also increases the convergence rate of the iteration. The Padè is a form of converting the analytical solution obtained through ADM method to polynomial rational form. The basic procedures are as follows:

1. Apply Laplace transform to the series solution Eq. (34) and setting:

$$
s=\frac{1}{t} .
$$

2. Apply Padè approximation to the solution from previous step to obtain the following approximation in the following rational form:

$$
\left[\frac{L}{M}\right]=\frac{P_{0}+P_{1} t+P_{2} t^{2}+\cdots P_{L} t^{L}}{q_{0}+q_{1} t+q_{2} t^{2}+\cdots q_{M} t^{M}} .
$$

and setting $t=\frac{1}{s}$.

3. Apply inverse Laplace transform on:

$$
\left[\frac{L}{M}\right]
$$

approximant.

\section{Application of Adomian-Padè method}

The accuracy of the ADM is improved using the principle of Laplace-Adomian-Padè method (MADM)

Apply Laplace transform to the series solution Eq. (34) as:

$$
\begin{aligned}
& \mathcal{L}[w(t)]=\frac{1}{s^{2}}-\frac{50202}{14521 s^{4}}+\frac{173212}{4493 s^{6}}-\frac{1268962}{9521 s^{8}}+\frac{1141417}{768 s^{10}}-\frac{1253711}{244 s^{12}}+\frac{15240749}{266 s^{14}}-\frac{13271621}{67 s^{16}} \\
& +\frac{55221251}{25 s^{18}}-\frac{45818617}{6 s^{20}}+\frac{255463610}{3 s^{22}}-\frac{294396257}{s^{24}}+\frac{3282837217}{s^{26}}-\frac{11349424550}{s^{28}} \\
& +\frac{126558379800}{s^{30}}-\frac{437537619900}{s^{32}}+\frac{4879018493000}{s^{34}}-\frac{16867742330000}{s^{36}}+\frac{188093601500000}{s^{38}} \\
& -\frac{650277183600000}{s^{40}}
\end{aligned}
$$


Also, replacing $s=\frac{1}{t}$, and calculating Padè approximant of $\left[\frac{5}{5}\right]$ and letting $t=\frac{1}{s}$, gives the following:

$$
\left[\frac{5}{5}\right]=\frac{\left(s^{2}-\frac{50202}{14521}\right)}{\left(s^{4}-\frac{173212}{4493}\right)}
$$

Applying the inverse Laplace transform to the Padè approximant of Eq. (39), the MADM solution is:

$$
w(t)=\frac{76381}{244507} \sin \left(\frac{50800 t}{20387}\right)+\frac{12871}{144730} \sinh \left(\frac{50800 t}{20387}\right),
$$

\section{Moment and stress}

The bending moment of $M_{x}$ and $M_{y}$ in $x$ and $y$ direction are:

$$
M_{x}=-D_{0}\left(\frac{\partial^{2} w}{\partial x^{2}}+v \frac{\partial^{2} w}{\partial y^{2}}\right),
$$

The surface stresses $\sigma x$ and $\sigma y$ in the $x$ and $y$ directions are:

$$
\sigma_{x}=6 \frac{M_{x}}{h^{2}},
$$

where $D_{0}$ is the flexural rigidity, $v$ is the Poisson's ratio and $w$ is the transverse deflection.

\section{Comparison of the results using numerical method}

Obtaining analytical solution for the natural frequency of this governing differential nonlinear Eq. (1) may be very difficult due to the nonlinear Winkler parameters introduced. Therefore, the next alternative approach is the numerical method. In this study, the finite difference method is employed for the discretization of the governing Eq. (3) along with the boundary conditions of Eqs.(4), (5) and (6). The rectangular plate illustrated in Fig. 2 is meshed along the spacexdivided into equally spaced $(\Delta x)$ points. The mesh points in the space are in $x_{1}, x_{2}, x_{3}, \ldots x_{N-1}, x_{N}$ where the edge nodes are $x_{1}$ and $x_{N}$, respectively.

$$
\Delta x=\frac{x_{N}-x_{0}}{N} \Rightarrow \frac{1-0}{11}=0.09
$$

For the validation of the analytical solutions obtained from the ADM, controlling parameters are set as 0 for validation. Investigating the effect of nonlinear Winkler parameter, nonlinear equation is formed and solved using the Jacobian matrix of Newton-Raphson method.

\section{Discretizing the governing equation}

$$
\begin{aligned}
& \frac{w_{i+2}-4 w_{i+1}+6 w_{i}-4 w_{i-1}+w_{i-2}}{(\Delta x)^{4}}-2 \lambda^{2} m^{2} \pi^{2}\left\{\frac{w_{i+1}-2 w_{i}+w_{i-1}}{(\Delta x)^{2}}\right\} \\
& -\left(\Omega^{2}+M_{a d d}-k_{L}-\lambda^{4} m^{4} \pi^{4}\right) w_{i}-k_{N L} w_{i}^{3}-g_{s}\left\{\frac{w_{i+1}-2 w_{i}+w_{i-1}}{(\Delta x)^{2}}\right\}=0,
\end{aligned}
$$

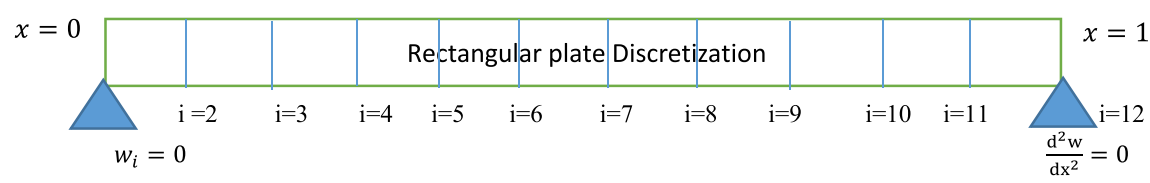

Fig. 2 Discretization of the thin rectangular plate into 12 nodes 
Having applied the finite difference approximation, grouping and rearranging the term, the final algebraic form of the finite difference equation becomes:

$$
\begin{aligned}
& w_{i+2}-4 w_{i+1}+6 w_{i}-4 w_{i-1}+w_{i-2}-2 \lambda^{2} m^{2} \pi^{2}(\Delta x)^{2}\left(w_{i+1}-2 w_{i}+w_{i-1}\right) \\
& -\left(\Omega^{2}+M_{a d d}-k_{L}-\lambda^{4} m^{4} \pi^{4}\right)(\Delta x)^{4} w_{i}+k_{N L}(\Delta x)^{4} w_{i}^{3}-g_{s}(\Delta x)^{2} \\
& \left(w_{i+1}-2 w_{i}+w_{i-1}\right)=0
\end{aligned}
$$

The discretization in Eq. (43) clearly shows that central difference approximation is employed in the discretization of the governing equation. Indirectly, the inner nodes $(i=2: N-1)$ will be utilized for solving the model. Therefore, only ten interior nodes will be used while the two node boundaries are excluded.

\section{Discretizing the boundary conditions}

For the sake of brevity, only the SS boundary condition is shown here, while the other boundary conditions SC and SF are solved following the same approach. The starting point and end are presented in the following unique form:

$$
\begin{gathered}
x=i, w_{i}=0, \frac{d^{2} W}{d X^{2}}=0 \Rightarrow \frac{w_{i+1}-2 w_{i}+w_{i-1}-v\left(m^{2} \pi^{2} \lambda^{2}\right) w_{i}}{(\Delta x)^{2}}=0, \\
i=1, w_{1}=0, w_{2}-2 w_{1}+w_{0}-v\left(m^{2} \pi^{2} \lambda^{2}\right) w_{1}=0 . \text { Therefore, } w_{2}=-w_{0} \\
x=i, w_{i}=0, \frac{d^{2} W}{d X^{2}}-v\left(m^{2} \pi^{2} \lambda^{2}\right) W=0 \Rightarrow \frac{w_{i+1}-2 w_{i}+w_{i-1}-v\left(m^{2} \pi^{2} \lambda^{2}\right) w_{i}}{(\Delta x)^{2}}=0, \\
i=12, w_{12}=0, w_{13}-2 w_{12}+w_{11}-v\left(m^{2} \pi^{2} \lambda^{2}\right) w_{12}=0, w_{13}+w_{11}=0 . \text { Therefore, } \\
\quad i \Rightarrow 2 \\
\quad w_{4}-\frac{28125 w_{3}}{6761}+\frac{42728 w_{2}}{6761}+w_{0}-\frac{\left(-639 \pi^{4}+639 \Omega^{2}\right) w_{2}}{9739369}=0 \\
i \Rightarrow 11
\end{gathered}
$$

Resolving the matrix yields the natural frequency.

\section{Results and discussion}

ADM is used in obtaining solution for free vibration analysis of a uniform thickness thin rectangular plate resting on elastic foundation and submerged in fluid. The accuracy of the analytical solutions obtained is validated with numerical and analytical solution of different method as reported in the cited literature [30] and presented in Table 1. Good agreement of the result is observed along the entire values under different boundary conditions. Generally, the natural frequency is expressed in dimensionless form $\Omega$. Comparing the analytical solution with the numerical shows that even for 5 iterations compared to 10 of numerical, the hybrid method still predicts better than the numerical, though the numerical has an edge handling the nonlinear term in the model. Results obtained with ADM is equally in good agreement compared to the analytical solution obtained by the reported literature [31] using DTM. The analytical solution by DTM converges at 21 iterations compared to $\mathrm{ADM}$ of 5 iterations. It is observed that the number of iterations needed to obtain convergence in relation to the natural frequency varies. For instance, fundamental mode requires 2-3 iterations for ADM while the second and third 


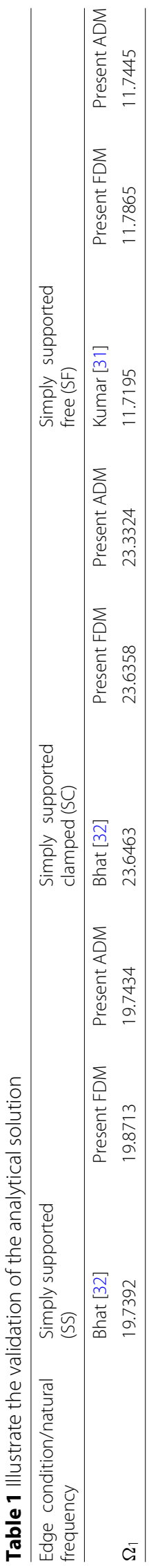




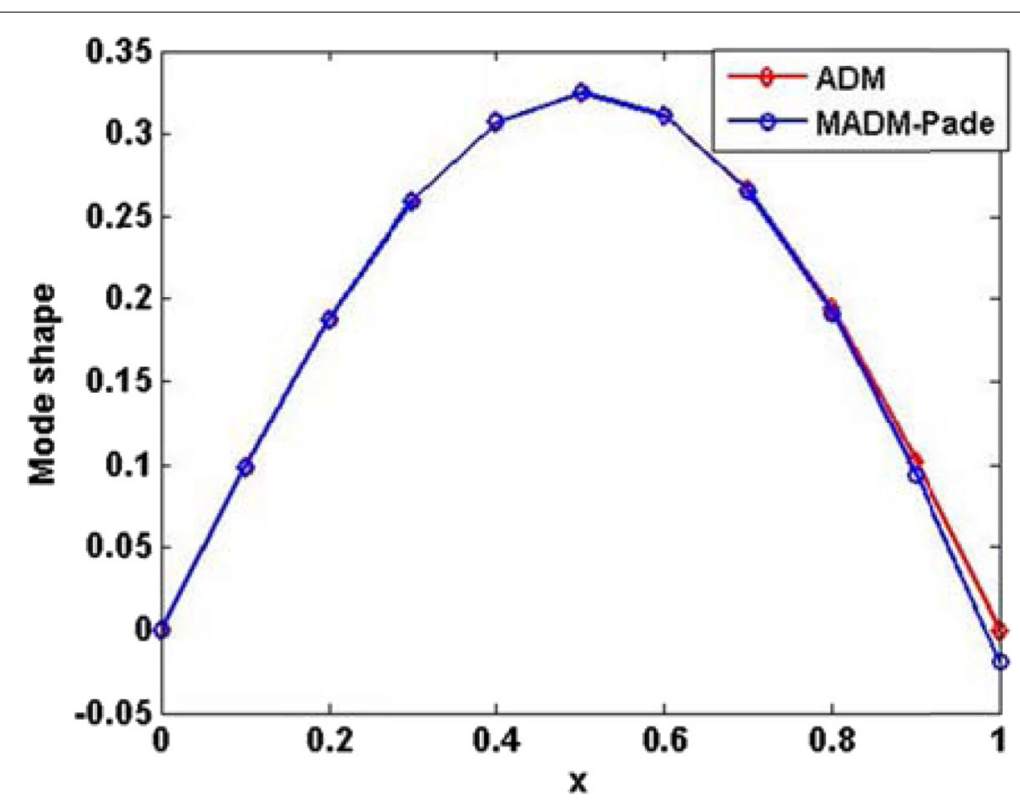

Fig. 3 Application of Laplace Padè approximant on ADM

iterations require more iterations. This phenomenon is attribute to more complex series function combination. The results shown in Table 1 demonstrate that, though fundamental natural frequency gives a reasonable prediction of the rectangular plate behaviour, more iterations are still required to give other higher mode natural frequencies and also increase the accuracy. It is interesting to note that present results agree very well with the past results.

Figure 3 illustrates the modification of the Adomian decomposition method by using the Laplace-Padè approximant approach. It is observed that MADM helps to improve the results most especially when the domain of the analysis is getting wider.

\section{Mode shapes}

The mode shape for the first two natural frequencies are shown in Fig. 4. It is important to note that the mode shape obeys the classical theory of vibration. For bending moment and stress, location of the vibrating node and antinodes are somehow different due to the vanishing mode of the boundary condition. Figures 4 and 5 show mode shape due to the bending moment and stress; it is clearly shown that the location of node and antinodes of
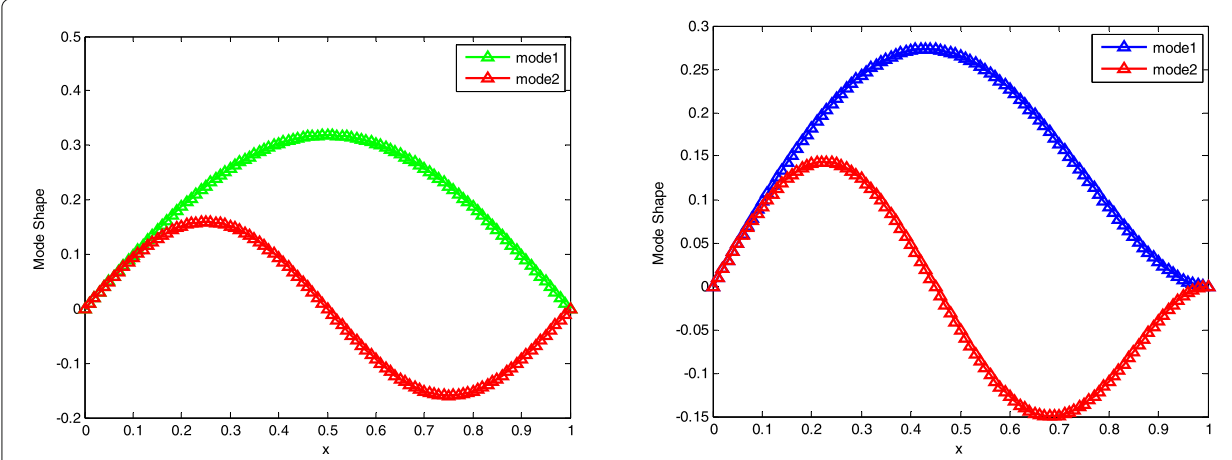

Fig. 4 Showing mode shapes of SS and SC boundary conditions 

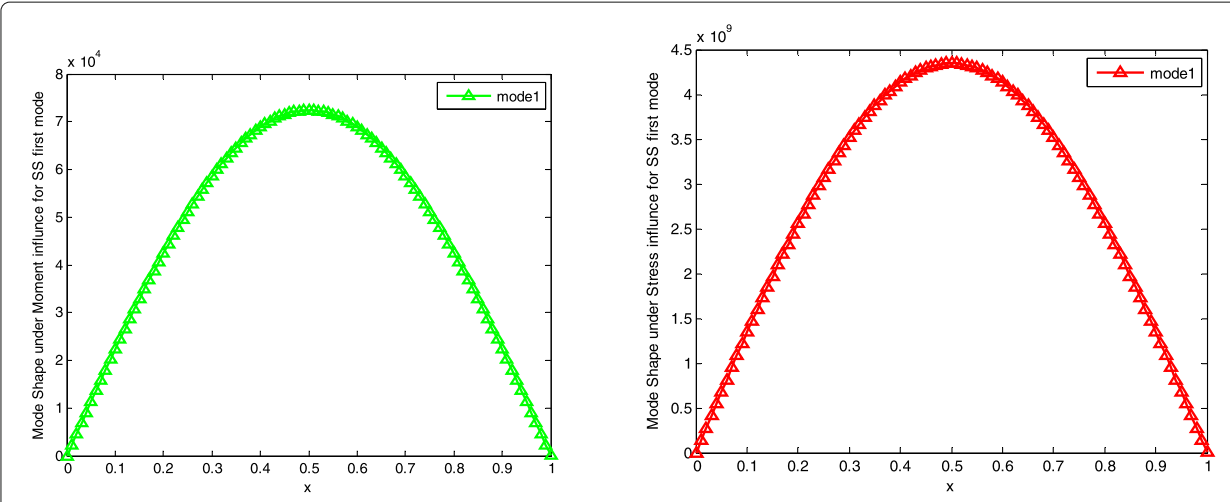

Fig. 5 Fundamental mode shape of SS plate under bending moment and stress respectively

the vibrating plate changes. While Fig. 6 depicts the extrema mode shape variation. It is clearly revealed that Figs. 5 and 6 when compared to Fig. 4 have different mode shapes. Invariably, the extrema mode shape location differs based on the boundary conditions. When the plate is submerged in fluid, the same mode shapes as illustrated in Fig. 4 are obtained; this is in accordance with the experimental result obtained by [33, 34]

\section{Variation of foundation parameter on natural frequency}

To investigate the variation effect of the elastic foundation on the first two natural frequencies of the thin rectangular plate under different boundary conditions discussed earlier, the material properties of the thin rectangular plate model are as follows [9]:

$E=207 \mathrm{Gpa}$, material density $\rho=7850 \mathrm{~kg} / \mathrm{m}^{3}$, thickness of the plate $h=0.01 \mathrm{~m}$ and density of the water $\rho=1000 \mathrm{~kg} / \mathrm{m}^{3}$. Thin rectangular plate is considered. Reservoir with a dimension of $5 \mathrm{~m} \times 5 \mathrm{~m} \times 5 \mathrm{~m}$ is used as a case study. Since dimensionless analysis is conducted, the results on natural frequencies obtained are valid for all thickness of the plate.

Table 2 illustrates the effects of the foundation parameter on natural frequency. In this case, consideration is given to (a) Elastic Winkler-type foundation $\left(g_{s}=0, k_{L}=\right.$ 10,50,100), (b) Elastic Pasternak-type foundation $\left(k_{L}=0, g_{s}=10,50,100\right)$ and (c) Two-parameter elastic foundation $\left(k_{L}=50, g_{s}=10,50,100\right)$

Parametric effect of nonlinear Winkler foundation is carried out as mentioned earlier using the finite difference method of numerical due to difficulty encountered when

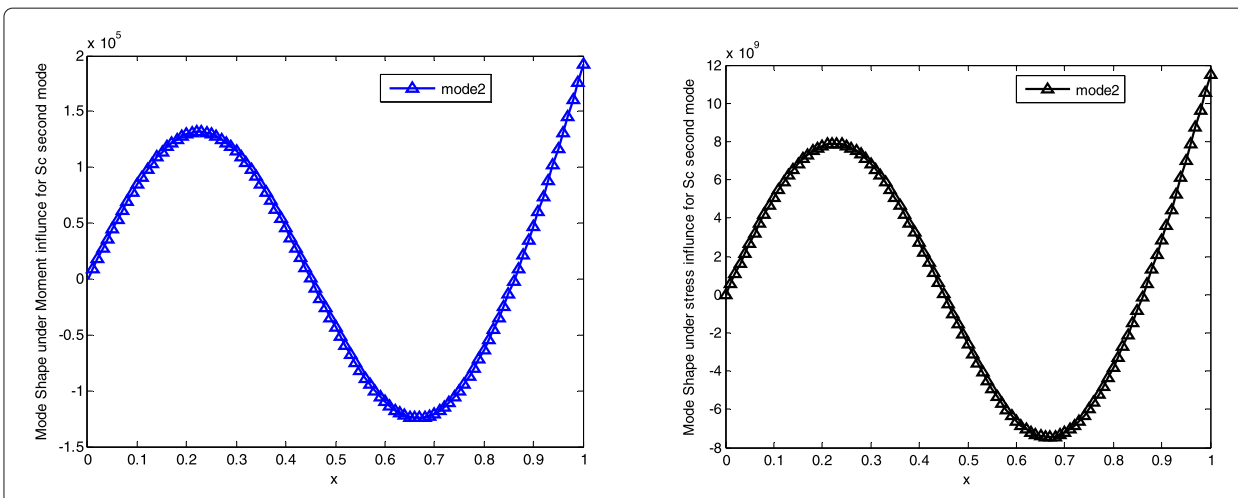

Fig. 6 Second mode shape of SC under moment and stress 
Table 2 Two-parameter foundation effect on first two natural frequencies of vibrating rectangular plate

\begin{tabular}{llllllll}
\hline Edge condition & $\begin{array}{l}\text { Natural } \\
\text { frequency }\end{array}$ & \multicolumn{2}{l}{$k_{\mathrm{L}}=50, \lambda=0.5$} & \multicolumn{3}{c}{$k_{\mathrm{L}}=50, \lambda=1.5$} \\
& & $g_{s}=10$ & $g_{s}=50$ & $g_{s}=100$ & $g_{s}=10$ & $g_{s}=50$ & $g_{s}=100$ \\
\hline SS & $\Omega_{1}$ & 17.3464 & 26.3758 & 34.4842 & 34.3159 & 39.653 & 45.4515 \\
& $\Omega_{2}$ & 46.9493 & 61.5091 & 75.8769 & 65.1907 & 76.3477 & 88.3341 \\
SC & $\Omega_{1}$ & 21.5364 & 30.1136 & 38.0430 & 37.2582 & 42.6778 & 48.5309 \\
& $\Omega_{2}$ & 56.5026 & 69.9870 & 83.7489 & 73.2330 & 84.0060 & 95.7134 \\
SF & $\Omega_{1}$ & 8.0257 & 7.0030 & 4.8888 & 24.7306 & 22.8481 & 19.1978 \\
& $\Omega_{2}$ & 22.8135 & 31.2448 & 39.1257 & 43.2468 & 48.6099 & 54.4463 \\
\hline
\end{tabular}

analysing with ADM. Although, it is a universal behaviour of plate to be affected by characteristic of elastic foundation, comparing Table 2 to Table 1 indicates that properly chosen value of foundation stiffness allows for both foundation stiffness and plate stiffness effect to be comparable.

It is clearly shown in all cases that increasing the foundation stiffness results into higher value of natural frequencies. Moreover, it is also observed that the effect of the difference in natural frequencies is more significant for higher mode of the vibrating rectangular plate. Figure 7 shows the graphical relationship of increasing foundation stiffness on the natural frequency. The graph depicts the linear relationship between stiffness increment and natural frequency.

\section{Variation of aspect ratio on natural frequency}

Considering the effect of change in the aspect ratio, recall that uniform thickness thin plate is the model. It is clearly seen that the reduction in the value of the aspect ratio (negative value) yields the same result as the positive variation of the aspect ratio increase in aspect ratio either in all cases results into increase in natural frequency of plate (Fig. 8). This phenomenon is a result of uniform thickness assumption in the model. The variation reduction in thickness has no effect.

\section{Submerging of plate in fluid}

Determining the effect of water in contact with plate involves incorporation of fluid pressure parameters into the model as stated earlier in the assumptions and using
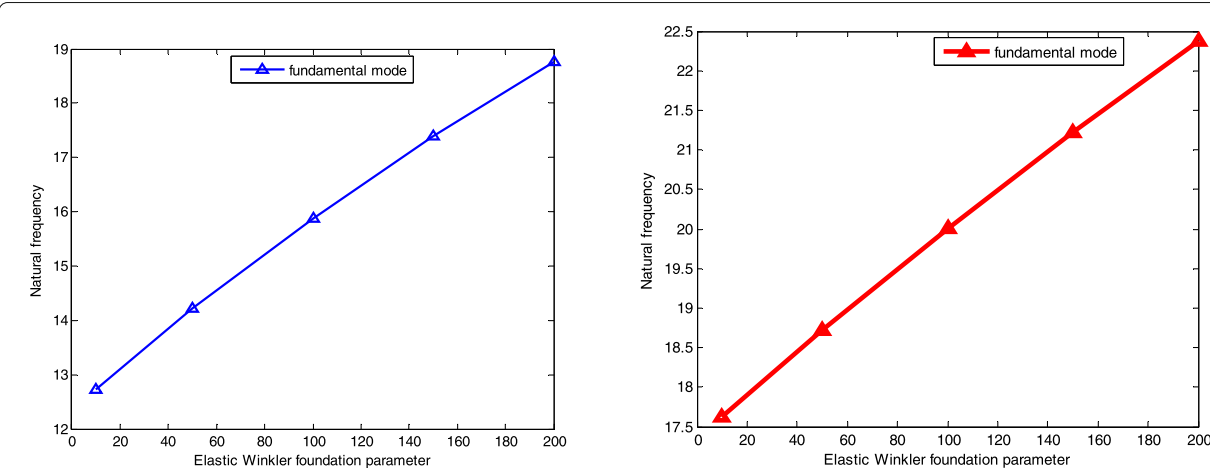

Fig. 7 Variation of foundation parameter on SS-edge and SC edge condition 

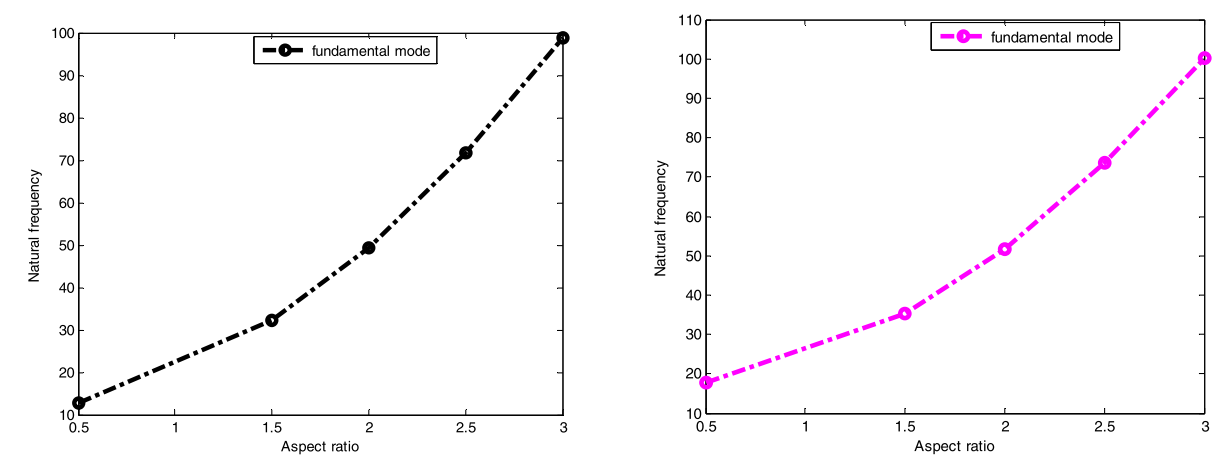

Fig. 8 Variation of aspect ratio on SS and SC edge condition

the parameters stated for the simulation. In order to illustrate the validity of the proposed analytical solutions, the numerical computation of the isotropic rectangular plate submerged in water is done and compared with those available from previous studies as shown in Table 3. The following parameters are adopted for the simulation according to [9].

$A=0.20165 \mathrm{~m}, B=0.65 \mathrm{~m}, h=0.963 \mathrm{~mm}$. Reservoir dimension $1.3 \mathrm{~m} \times 0.55 \mathrm{~m} \times 0.8 \mathrm{~m}$.

Water has a noticeable effect on the characteristic behaviour of plate in contact with fluid. Table 4 depicts the natural frequency of plate when submerged and immersed in fluid. The fluid under consideration here is water; it is globally known that natural frequency becomes lower when the vibrating plate is in contact with fluid. The behaviour is referred to as added virtual mass effect. The behaviour is the result of the transfer of vibration by plate in contact with water in motion thereby increasing the kinetic energy of the total system. It is found that the presence of water has most significant effect on the fundamental mode regardless of the boundary condition of the rectangular plate.

\section{Conclusion}

In this study, the investigation of dynamic response of isotropic rectangular plates resting on Winkler and Pasternak foundations is presented. The coupled governing nonlinear partial differential equation is transformed to nonlinear ordinary differential equation using the Galerkin method of separation. The coupled nonlinear ordinary differential equations have been solved using the Adomian decomposition method. Moments and stress are determined. The accuracies of the obtained analytical solutions were ascertained with the numerical and analytical methods reported in the literature, and the results are confirmed to be in good agreement. The obtained analytical solutions were used to examine the effects of foundation parameter, aspect ratio and presence of fluid in contact with plate. From the parametric studies, the following observations were established. Increases in the elastic foundation parameter increases the natural frequency. Increases in aspect ratio increases the natural frequency. Submerging the plate in fluid

Table 3 Validation of solution for fluid-plate interaction

\begin{tabular}{llll}
\hline \multicolumn{2}{l}{$\begin{array}{l}\text { Natural frequencies of simply supported plate submerged in fluid } \\
\text { Mode }\end{array}$} & Present & Percentage error \\
\hline 1 & 28.72 & 22.118 & 22 \\
2 & 117.725 & 108.993 & 7 \\
3 & 154.51 & 156.596 & 1.35 \\
\hline
\end{tabular}




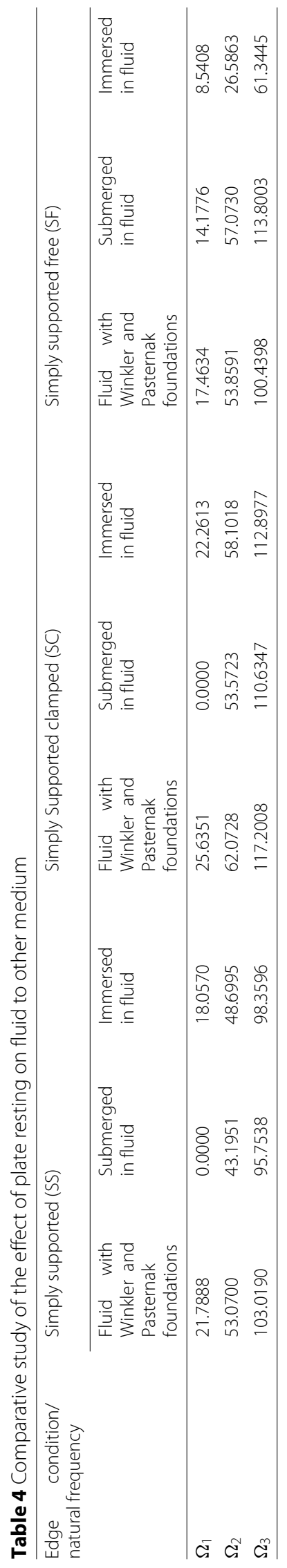


lowers the natural frequency. "The major benefit of ADM is the ease of application to various types of differential and integral equations, linear or nonlinear, homogeneous or inhomogeneous, with constant coefficients or with variable coefficients; likewise, it is capable of greatly reducing the size of computational work while still maintaining high accuracy of the numerical solution. [36].

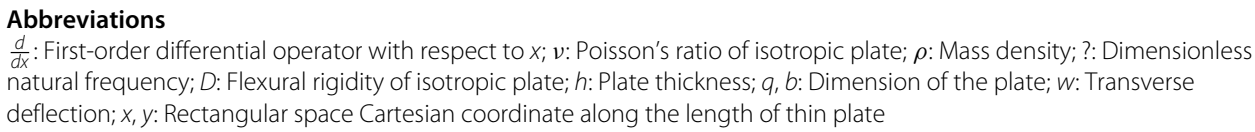

\section{Competing interests}

The authors declare that they have no competing interests.

\section{Author details}

${ }^{1}$ Department of Mechanical Engineering, University of Lagos, Akoka, Lagos, Nigeria. ${ }^{2}$ Department of civil and Environmental Engineering, University of Lagos, Akoka, Nigeria.

Received: 5 April 2019 Accepted: 5 February 2020

Published online: 29 April 2020

\section{References}

1. Motaghian, S., Akin, J. E.: On the free vibration response of rectangular plates, partially supported on elastic foundation. Appl. Math Model. 36(9), 4473-4482 (2012)

2. Rezaiefar, A., Galal, K.: Free vibration of thin rectangular steel plates with geometrically nonlinear load-displacement behaviour. Thin-Walled Struct. 129, 381-390 (2018)

3. Shariyat, M., Alipour, M. M.: Differential transform vibration and modal stress analyses of circular plates made of two-directional functionally graded materials resting on elastic foundations. Arch Appl. Mech-Springer-Verlag. 81, 1289-1306 (2011)

4. Benferhat, R., Dao-Tahar, H. D., Mansor, M. S., Hadji, L.: Effect of porosity on the bending and free vibration response of functionally graded plate resting on Winkler-Pasternak foundation. Earthq. Struct., 1429-1449 (2016). https://doi. org/10.12989/eas.2016.10.6.1429

5. Lamb: On the vibration of an elastic plate in contact with fluid. Proc. R. Soc. Ser. A Lond. 98, 205-216 (2016)

6. Sobamowo, G., Jayeisinmi, L., Oke, D., Yinusa, A., Adedibu, O.: Unsteady casson nanofluid squeezing flow between two parallel plates embedded in a porous medium under the influence of magnetic field. Open J. Math. Sci. 3(1), 59-73 (2019). https://doi.org/10.30538/oms.2019.0049.MSC:76A05

7. Kwak, M. K.: Vibration of circular plate in contact with water. Trans. ASME. 58, 480-483 (1991)

8. Soedel, S. M., Soedel, W.: On the free and forced vibration of a plate supporting a free sloshing surface liquid. Int. J. Sound Vib. 171(2), 159-171 (1994)

9. Kerboua, Y., Lakis, A. A., Thomas, M., Marcouiller, L.: Vibration analysis of rectangular plates coupled with fluid. Appl. Math Model. 32, 2570-2586 (2008). https://doi.org/10.1016/j.apm.2007.09.004

10. Ozdemir, Y. I: Forced vibration analysis of Mindlin plates resting on Winkler foundation. Chall. J. Struct. Mech. 4(1) (2018). https://doi.org/10.20528/cjsmec.2018.01.004

11. Mohd, S., Umair, N. P., Gulhane, A., Al-Robaian, R. A., Khan, S. A.: On numerical investigation of semi-empirical relations representing local Nusselt number at lower nozzle-target spacings. Int. J. Eng. 32(1), 137-145 (2019)

12. Nikbakhat, R., Behnamfar, F.: Response spectra of structures under subway induced vibrations. Int. J. Eng. 31(12), 2009-2015 (2018)

13. Bayat, M., Pakar, I., Bayat, M.: Analytical study on vibration frequencies of tapered beams. Lat. Am. J. Solids Struct. 8, 149-162 (2011)

14. Werfalli, N. M., Aburuga, T. K.: Galerkin finite element analysis for bending of anisotropic plates. Fourth Arab. Conf. Materi. Sci. ACMS Tripoli Libya. 4(1), 319-331 (2005)

15. Zingil, M., Topal, F. S.: Oscillation criteria for nonlinear dynamic equations on time scale. Open J. Math. Sci. 2(1), 307-322 (2018). http://dx.doi.org/10.3053810ms2018.037.MSC34k11,39A10,39A99 
16. Rehman, H., Saheem, M. S., Ahmad, A.: Combined of Homotopy perturbation method (HPM) and double Sumudu transform to solve fractional KDV equations. Open J. Math. Sci. 2(1), 29-38 (2018). http://dx.doi.org10.30538/ oms2018.0015

17. Yazdi, A. A.: Assessment of homotopy perturbation method for study the forced nonlinear vibration of orthotropic circular plate on elastic foundation. Lat. Am. J. Solid Struct. 13, 243-256 (2016)

18. Bambill, D. V., Rossit, R. E., Laura, R. E.:Transverse vibration of an orthotropic rectangular plate of linearly varying thickness and with a free edge. J. Sound Vib. 235(3), 530-538 (2000)

19. Adomian, G., Rach. R.: Nonlinear stochastic differential delay equations. J. Appl. Anal. Math. 91, 94-101 (1983)

20. Dehghan, M., Hamidi, A., Shakourifar, M.: The solution of coupled Burgers' equations using Adomian-Pade technique. Appl. Math. Comput. 189(2), 1034-1047 (2007). https://doi.org/10.1016/j.amc.2006.11.179

21. Tatari, M., Dehghan, M.: The use of the Adomian decomposition method for solving multipoint boundary value problems. Phys. Scr. 73(6), 672-676 (2006). https://doi.org/10.1088/0031-8949/73/6/023

22. Dehghan, M., Tatari, M.: The use of Adomian decomposition method for solving problems in calculus of variations. Math. Probl. Eng. 2006, 1-12 (2006). https://doi.org/10.1155/MPE/2006/65379

23. Dehghan, M., Tatari, M.: Finding approximate solutions for a class of third-order non-linear boundary value problems via the decomposition method of Adomian. Int. J. Comput. Math. 87(6), 1256-1263 (2010). https://doi.org/10.1080/ 00207160802270853

24. Dehghan, M., Salehi, R.: A semi-numeric approach for solution of the Eikonal partial differential equation and its applications. Numer. Methods Partial Differ. Equat. 26(3), 702-722 (2010). https://doi.org/10.1002/num.20482

25. Dehghan, M., Shakeri, F.: The use of the decomposition procedure of Adomian for solving a delay differential equation arising in electrodynamics. Phys. Scr. 78(6), 11 (2008)

26. Al-Zhour, Z., Al-Mutairi, N., Alrawajeh, F., Alkhasawneh, R.: Series solutions for the Laguerre and Lane-Emden fractional differential equations in the sense of conformable derivative. Alex. Eng. J. In press, Online, 1-8 (2019). http://dx.doi.10.1016/j.aej.2019.11.012

27. Al-Zhour, Z., Alrawajeh, F., Al-Mutairi, N., Alkhasawneh, R.: New results on the conformable fractional Sumudu transform: theories and applications. Int. J. Anal. Appl. 17(6), 1019-1033 (2019). https://doi.org/10.28924/2291-863917-2019-1019

28. Leissa, A. W.: Vibration of plates, Columbus. Scientific and Technical information Division, Office of Technology Utlilization National Aeronautics and Space Adminstration, Ohio (1969)

29. Chakraverty, S.: Vibration of plate. CRC Press Taylor \& Francis Group, London (2009)

30. Dumir, P. C.: Non-linear vibration and postbuckling of orthotropic thin circular plates on elastic foundations. Appl. Acoust. 19, 401-419 (1986)

31. Kumar, Y.: Free vibration analysis of isotropic rectangular plates on winkler foundation using differential transform method. Int. J. Appl. Mech. Eng. 18(2), 589-597 (2013). https://doi.org/10.2478/ijame-2013-0036

32. Bhat, R. B.: Natural frequencies of rectangular plates using characteristic orthogonal polynomials in Rayleigh-Ritz method. J. Sound Vib. 102, 493 (1985). https://doi.org/10.1016/S002-460X(85)80109-7

33. Espinosa, F. M., Gallego-Juarez, J. A.: On the resonance frequencies of water loaded circular plates. J. Sound Vib. 94(2), 217-222 (1984)

34. Kwak, M. K., Kim, K. C.: Axisymmetric vibration of circular plate in contact with fluid. J. Sound Vib. 146, 381-389 (1991)

35. Haddara, M. R., Cao, S.: A study of the dynamic response of submerged rectangular flat plates. Mar. Struct. $\mathbf{9}(10)$, 913-933 (1996). https://doi.org/10.1016/0951-8339(96)00006-8

36. Wazwaz, A.: Adomian decomposition method for a reliable treatment of the Emden Fowler equation. Appl. Math. Comput. 161, 543-560 (2005)

37. Jiao, Y. C., Yamamoto, Y., Dang, C., Hao, Y.: An aftertreatment technique for improving the accuracy of Adomian's decomposition method. Comput. Math. Appl. 43, 783-798 (2002)

\section{Publisher's Note}

Springer Nature remains neutral with regard to jurisdictional claims in published maps and institutional affiliations.

\section{Submit your manuscript to a SpringerOpen ${ }^{\circ}$ journal and benefit from:}

- Convenient online submission

- Rigorous peer review

- Open access: articles freely available online

- High visibility within the field

- Retaining the copyright to your article

Submit your next manuscript at $\boldsymbol{\triangleright}$ springeropen.com 